\title{
Article \\ Using a Human Circulation Mathematical Model to Simulate the Effects of Hemodialysis and Therapeutic Hypothermia
}

\author{
Jermiah J. Joseph ${ }^{1,2} \mathbb{D}$, Timothy J. Hunter ${ }^{1,2} \mathbb{D}$, Clara Sun ${ }^{1,2}$, Daniel Goldman ${ }^{2}$, Sanjay R. Kharche ${ }^{1,2,3, *(\mathbb{D})}$ \\ and Christopher W. McIntyre ${ }^{1,3, *}$
}

1 Lawson Health Research Institute, 750 Baseline Road East, London, ON N6C 2R5, Canada; jjosep56@uwo.ca (J.J.J.); thunte27@uwo.ca (T.J.H.); csun286@uwo.ca (C.S.)

2 Department of Medical Biophysics, Western University, 1151 Richmond Street North, London, ON N6A 5C1, Canada; dgoldma2@uwo.ca

3 Room ELL126C, Kidney Clinical Research Unit, Lawson Health Research Institute, Zone E, Victoria Hospital, 800 Commissioners Road East, London, ON N6A 5W9, Canada

* Correspondence: Sanjay.Kharche@lhsc.on.ca (S.R.K.); Christopher.McIntyre@lhsc.on.ca (C.W.M.); Tel.: +1-519-685-8500 (ext. 56047) (S.R.K.); Fax: +1-519-685-8072 (S.R.K.)

Featured Application: Human circulation and dialysis 0D model.

check for updates

Citation: Joseph, J.J.; Hunter, T.J.; Sun, C.; Goldman, D.; Kharche, S.R.; McIntyre, C.W. Using a Human Circulation Mathematical Model to Simulate the Effects of Hemodialysis and Therapeutic Hypothermia. Appl. Sci. 2022, 12, 307. https://doi.org/ 10.3390/app12010307

Academic Editor: Elena G.

Govorunova

Received: 7 December 2021

Accepted: 22 December 2021

Published: 29 December 2021

Publisher's Note: MDPI stays neutral with regard to jurisdictional claims in published maps and institutional affiliations.

Copyright: (c) 2021 by the authors. Licensee MDPI, Basel, Switzerland. This article is an open access article distributed under the terms and conditions of the Creative Commons Attribution (CC BY) license (https:// creativecommons.org/licenses/by/ $4.0 /)$.

\begin{abstract}
Background: We developed a hemodynamic mathematical model of human circulation coupled to a virtual hemodialyzer. The model was used to explore mechanisms underlying our clinical observations involving hemodialysis. Methods: The model consists of whole body human circulation, baroreflex feedback control, and a hemodialyzer. Four model populations encompassing baseline, dialysed, therapeutic hypothermia treated, and simultaneous dialysed with hypothermia were generated. In all populations atrial fibrillation and renal failure as co-morbidities, and exercise as a treatment were simulated. Clinically relevant measurables were used to quantify the effects of each in silico experiment. Sensitivity analysis was used to uncover the most relevant parameters. Results: Relative to baseline, the modelled dialysis increased the population mean diastolic blood pressure by $5 \%$, large vessel wall shear stress by $6 \%$, and heart rate by $20 \%$. Therapeutic hypothermia increased systolic blood pressure by $3 \%$, reduced large vessel shear stress by $15 \%$, and did not affect heart rate. Therapeutic hypothermia reduced wall shear stress by $15 \%$ in the aorta and $6 \%$ in the kidneys, suggesting a potential anti-inflammatory benefit. Therapeutic hypothermia reduced cardiac output under atrial fibrillation by $12 \%$ and under renal failure by $20 \%$. Therapeutic hypothermia and exercise did not affect dialyser function, but increased water removal by approximately $40 \%$. Conclusions: This study illuminates some mechanisms of the action of therapeutic hypothermia. It also suggests clinical measurables that may be used as surrogates to diagnose underlying diseases such as atrial fibrillation.
\end{abstract}

Keywords: lumped parameter model; human circulation; dialysis; ordinary differential equations; sensitivity analysis

\section{Introduction}

\subsection{Clinical Motivation}

Chronic kidney disease affects a significant proportion of the Canadian and worldwide elderly patient population [1]. Hemodialysis is a repetitive treatment that prolongs the lives of the patients, but causes deleterious side effects [2]. Dialysis promotes cardiac stunning [3], accumulative ischemic damage to multiple organs [4,5], and an altered coronary blood flow [6]. Dialysis is also known to promote cardiac arrhythmogenicity [7]. Our recent clinical-imaging-modelling study strongly suggests that dialysis is a causal factor in augmenting organ level blood flow heterogeneity [6], altered hepatic and renal blood flow [4], and affects cerebral circulation. In addition, dialysis is a resource intensive treatment that is 
a significant financial burden on health care systems [8]. Treatments such as therapeutic hypothermia may reduce the severity of the dialysis induced side effects as seen in large scale clinical studies [9], as well as observed in animal models [10]. An augmented understanding of the mechanisms of hemodialysis under prevalent co-morbidities may assist to the translation of our clinical therapeutic hypothermia research into clinical practice.

\subsection{Overview of Past Modelling}

Blood flow modelling is an advanced field that is now routinely used to complement clinical research. Multi-scale models are increasingly being used to quantitatively support clinical decision making [11]. This preliminary study deploys a reduced order lumped parameter (0D) model of the human circulation.

A human circulation model $[12,13]$ was used in our recent study where arterial stiffening was found to be a prime cause of hypertension [14]. A more detailed and calibrated whole body human model $[15,16]$ has been deployed widely, including to predict the consequences of orthostatic stress and exercise [17]. The relative contribution of dialyzer membrane related convection and diffusion have been assessed using steady state hemodynamic modelling [18]. A spatially extended description of bicarbonate dialysis demonstrates the potential for model personalization [19]. Other studies have evaluated the benefit of citrate in calcium dialysate profiling [20] or dialysate sodium profiling [21]. The primary end point of water removal from the patient in a hemodialysis operation was modelled to identify candidate factors that can be personalized [22,23]. Modelling has been used to demonstrate mechanisms of solute removal [24] and uremic toxin removal [25] in typical simulated dialysis procedures. The procedure of dialysis itself has been modelled by Ursino et al. [26], which forms a sub-model in the presented study.

This study extends prior reduced order human circulation modelling by simulating patient populations, extending the description of the kidneys to two parallel circulation components, and assesses the modelling in light of multiple disease conditions.

\subsection{Study Aims}

This work provides an in silico system for a preliminary qualitative assessment of dialysis treatment and therapeutic hypothermia therapy. The presented model was constructed to provide a skeleton in silico model, an approach that may limit its functionality. Specifically, our aims were to:

(a) construct a composite model consisting of three sub-models from the literature that are coupled to each other: a whole body human circulation model [16], a baroreflex model [27], and a dialyzer model [26];

(b) use the composite model to simulate clinically relevant outputs in four populations consisting of baseline, dialysis, therapeutic hypothermia, and dialysis with therapeutic hypothermia. As such, the baseline population is treated as control while all other populations are considered to be under pathological conditions;

(c) simulate the changes in model generated clinical outputs under atrial fibrillation as a prevalent co-morbidity, and under exercise as a potential treatment that alleviates the side effects of dialysis; and

(d) to perform a sensitivity analysis to identify the important parameters in our model.

The paper is organized as follows (see Figure 1). Section 2 details the methods used in this study with Section 2.1 describing the model components, Section 2.2 describing the four model populations used, Section 2.3 describing the clinically relevant model outputs used to evaluate each experiment, Section 2.4 describing the atrial fibrillation and exercise simulations, Section 2.5 describing how parametric sensitivity was assessed, and Section 2.6 describing our numerical methods. Section 3 describes the results of the study with Section 3.1 describing the baseline (control) model and Section 3.2 describing in greater detail the clinically relevant model outputs. The roles of atrial fibrillation, exercise, right homogenous kidney dysfunction and heterogenous right kidney dysfunction are detailed in Sections 3.3-3.6. Section 3.7 describes the effectiveness of volume removal under diseased 
conditions and Section 3.8 describes the most significant model parameters. Section 4 describes the study conclusions and discussions with a subsection describing the study limitations. The supplement provides additional details that are desirable to support this work.

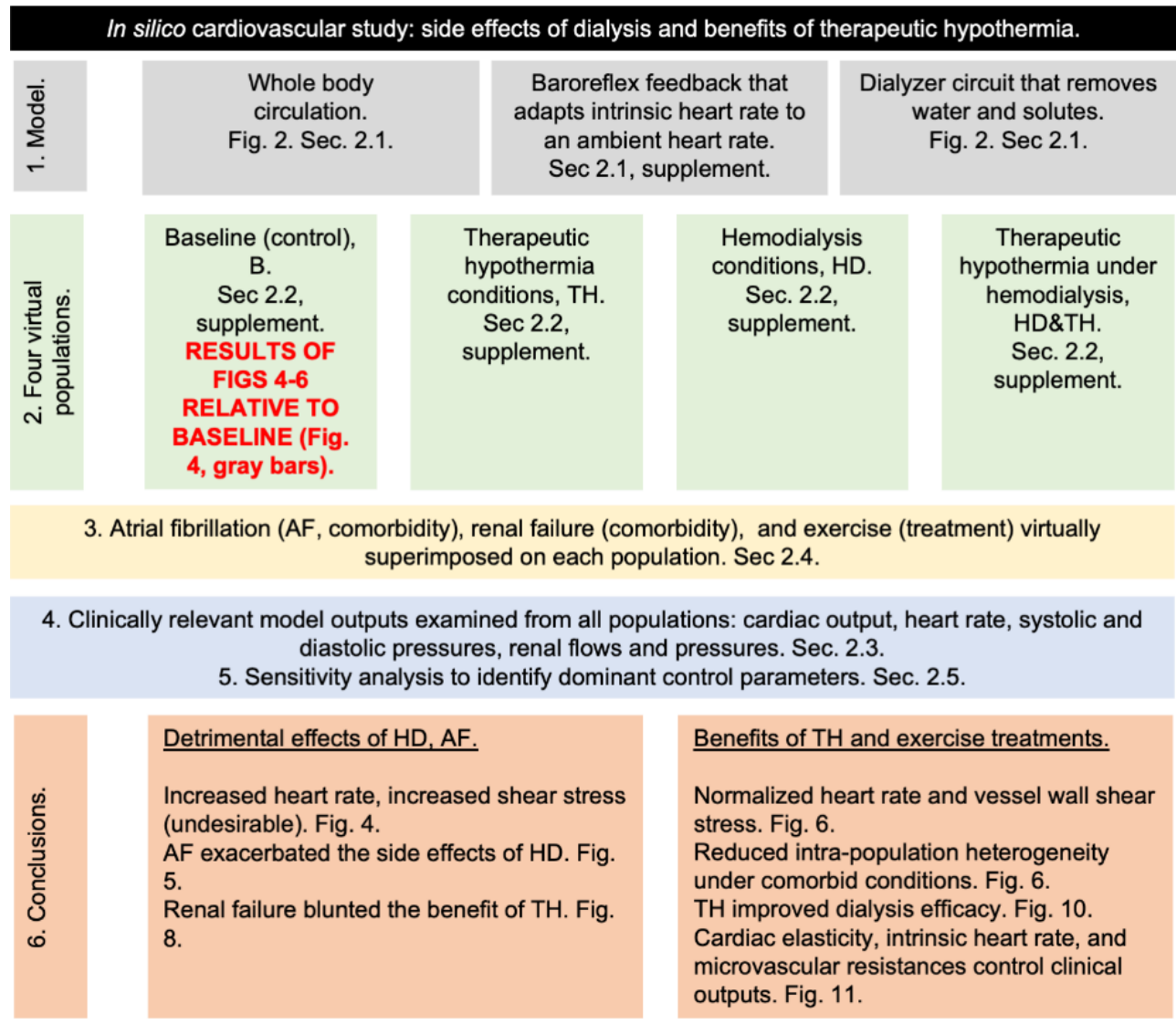

Figure 1. Block diagram summary of the presented work with sign posting to relevant sections.

\section{Methods}

\subsection{Model Components}

The composite model consists of a whole body human circulation model where baroreflex control is present, which is coupled to a representation of a dialysis machine. A whole body human circulation model [15,16] with known baroreflex control mechanisms [27-29] was coupled to a virtual dialysis unit [26,30] (see Figure 2 and Figures S1-S3). Model parameters were inherited from the respective calibrated parent sub-models [15,26,27]. The composite circulation model was extended to include detailed kidneys. The two detailed kidneys, left and right, were placed in parallel and both received inlet flow from the descending aorta. Further, renal microvasculature was represented within each kidney using six resistance-capacitance-resistance Windkessel representations in parallel (Supplementary Figure S1). The three element Windkessel model consists of a proximal resistance element which represents the impedance of the artery, a capacitor which represents the compliance of the elastic artery, and a distal resistor to represent the resistance of the downstream microvasculature. Each renal microvascular bed was assigned resistance and capacitance values derived from the parent model [15]. 
A. Whole body circulation model.

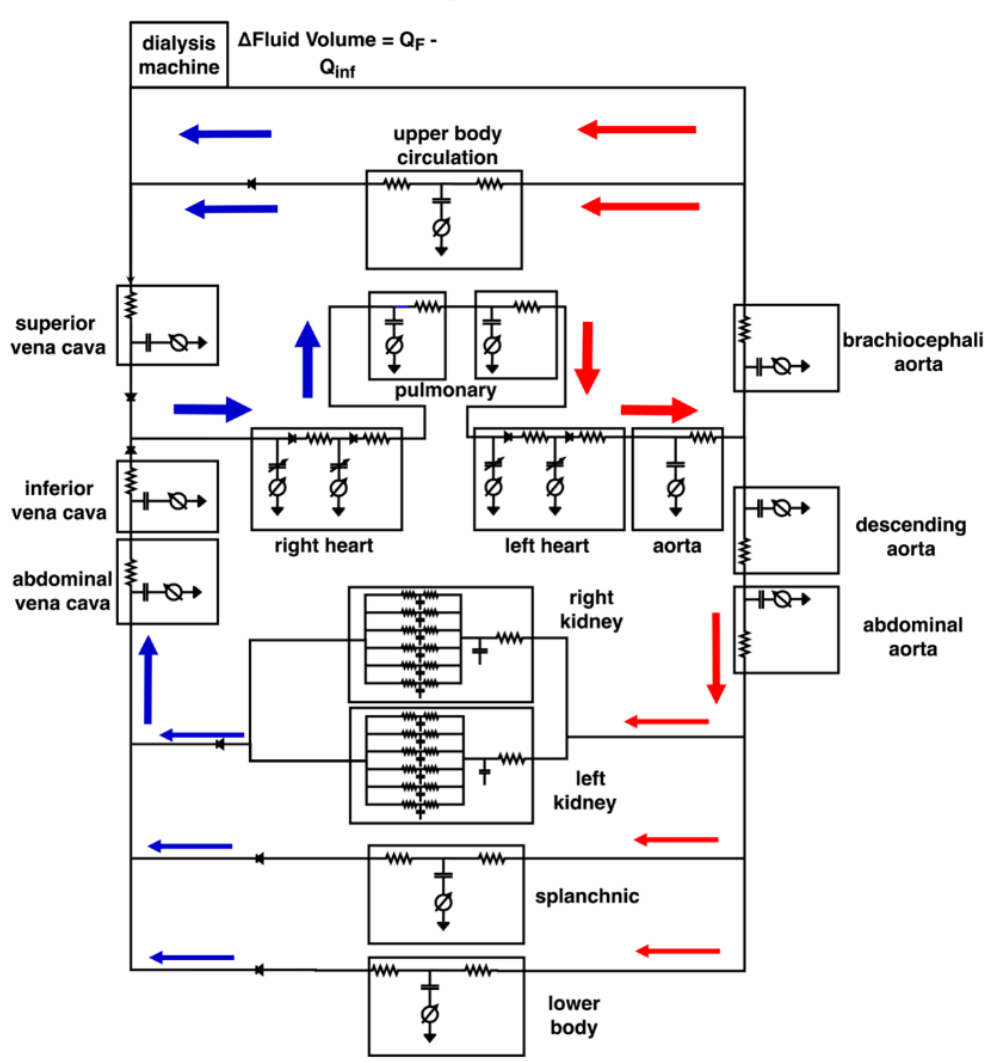

B. Symbols used.

\begin{tabular}{cc} 
Symbol description (electrical analogue). & Symbols. \\
\hline Vessel resistance (resistance). & -1 \\
\hline Vessel elasticity (capacitance). & - \\
\hline Vessel variable elasticity (variable capacitance). & $-Q-$
\end{tabular}

Figure 2. Schematic of presented model. (A) Electrical circuit analogue of the reduced order whole body human circulation model. Arrows represent direction of blood flow in arteries (red) and veins (blue). The hemodynamic symbols and their electrical analogues are shown at the bottom of the figure in panel (B). Symbols used in panel (A). Acronyms: $Q_{\mathrm{f}}$ : ultrafiltration rate. $Q_{\text {inf }}$ : injecting flow rate of the dialyser fluid.

A virtual hemodialysis system [26,30] was incorporated to model the impact of dialysis as a clearance of solutes (toxins and water). The implementation of hemodialysis consists of three fluid compartments and two solute compartments, as well as the dialyzer unit. The fluid compartments represent intracellular, extracellular and plasma fluid volume, while the solute compartments represent the amount of intracellular and extracellular (including plasma) solutes. Flux between the compartments is governed by osmosis (fluids) and diffusion (solutes) through membranes. Similarly, solute clearance is governed by diffusive transport between ultrafiltrate $\left(\mathrm{Q}_{\mathrm{F}}\right)$ and blood flowing through the dialyzer $\left(\mathrm{Q}_{\mathrm{B}}\right)$, as described by Ursino et al. [26]. The ultrafiltration rate, UF, was assumed to be $0.5 \mathrm{~L} / \mathrm{h}$ as in previous studies. Notably, the main influence of the dialyzer on the system is a removal of toxins and water. The presented model assumes a controllable ultra-filtration rate as an experimental parameter. As such, the model assumes high levels of toxins and water in the blood as compared to the dialysate, and initial conditions can be found in Lim et al. [30]. Whereas the dialyzer alters blood composition, the cardiovascular system was assumed not to influence the dialyzer.

A baroreflex mechanism was implemented to model sympathetic and parasympathetic control of cardiac function in response to mean arterial pressure [28,31]. The baroreflex 
implementation is a system of first order filters that takes mean arterial pressure as an input, and outputs adjustment coefficients to the baseline values for cardiac elastances as well as a " $\Delta$ " HR term which is added to the intrinsic heart rate to obtain the baroreflex controlled heart rate.

\subsection{Four Model Populations}

Models populations for baseline (B), therapeutic hypothermia (TH), hemodialysis (HD), and hemodialysis with therapeutic hypothermia (HD\&TH) were simulated in this study. The baseline population devoid of dialysis or therapeutic hypothermia was taken as the control (also called baseline) to which the behaviour of all other pathological cases was compared.

Each model population consisted of $10^{4}$ instances. To generate model instances, a number of model parameters were perturbed to represent inter-patient variability. Specifically, all large resistances, all large compliances, the four cardiac elastances, the intrinsic heart rate, and sympathetic feedback strength enlisted in Supplementary Materials, Table S1, were perturbed simultaneously. Model parameters values were randomly sampled from Gaussian distributions with predefined mean values and a coefficient of variation of 0.25. An implementation of the Box-Muller algorithm [32] ensured Gaussian sampling, while representation through the parameter range was ensured by use of Latin Hypercube Sampling [33].

In all simulations, four cases including baseline (represented by B), hemodialysis (represented by HD), therapeutic hypothermia (represented by TH), and hemodialysis under therapeutic hypothermia (represented by HD\&TH) were considered. The first of the four model populations were generated under baseline conditions based upon parameter values inherited from the parent sub-models. The HD population was generating by activating clearance of carbonate, urea, and small molecules (potassium, calcium, and sodium) from the blood [26]. Further, HD induced increase in the intrinsic heart rate to $90 \mathrm{bpm}$ was implemented (Burton et al., 2009). In addition, under HD conditions, ultrafiltration from blood to the dialyzer was activated. Therapeutic hypothermia (TH) treatment was simulated by implementing an experimentally observed $\mathrm{Q}_{10}$ scaling of all model resistances [34]. Whereas physiological temperature was taken to be $37.5^{\circ} \mathrm{C}$, $\mathrm{TH}$ temperature was conservatively assumed to be $35.5 \mathrm{C}$ throughout the body. These values for physiological temperature and $\mathrm{TH}$ used for this model study are a representative parameter for physiological core temperature. The following relationship was used to scale all blood vessel diameters with respect to temperature:

$$
D_{35.5}=D_{37.5} \times Q_{10}^{\beta(35.5-37.5)}
$$

where $D_{\mathrm{T}}$ represents vessel diameter at temperature T, $Q_{10}=2.96$, and $\beta=0.08$ [34]. The diameters were then used to re-assign all resistance values in the model. Finally, HD\&TH conditions simulated whole body cooling of $2 \mathrm{C}$ simultaneous to HD as describe above.

\subsection{Clinically Relevant Model Outputs}

Clinically relevant outputs were obtained in all simulation experiments. The outputs were cardiac output, heart rate, systolic and diastolic pressures of the systemic artery (aorta), renal pressures and flows. In addition, time variations of all dynamic quantities were recorded for further processing. Shear as indicator of myogenic stimulation [35] was computed in all blood vessels, and recorded in the aorta (low resistance and high compliance) and kidney microvasculature (high resistance). Wall shear stress was estimated using the flow $(Q)$, diameter $(D)$, and blood viscosity $(\eta)$ as [36]:

$$
\tau=\frac{4 \eta Q}{\pi(D / 2)^{3}}
$$


where $\tau$ is the wall shear stress, $\eta=3 \times 10^{-5} \mathrm{mmHg}-\mathrm{s}$ is the constant blood viscosity in all vessels [15], $Q$ is flow in the vessel, and $D$ is the temperature dependent diameter.

\subsection{Simulated Atrial Fibrillation and Exercise Conditions}

In this work, the effects of atrial fibrillation (AF), exercise, and right kidney failure on the efficacy of $\mathrm{HD}$ and TH were assessed.

AF was simulated by clamping both atrial elastances to their diastolic values. The diastolic value of elastance in the left atrium is $0.5 \mathrm{~mL} / \mathrm{mmHg}$, and in the right atrium is $0.3 \mathrm{~mL} / \mathrm{mmHg}$. In addition, AF induced irregular heart rate was incorporated into the model simulated as described in the literature [37].

Exercise was simulated by increasing the ventricular end-systolic elastances and the heart rate, while reducing compartment resistances and large vessel compliances [37]. Specifically, the ventricular systolic elastances were increased by $140 \%$, and the intrinsic heart rate was increased by $28.6 \%$. Simulated exercise also encompassed a reduction of all microvascular resistances by $33 \%$, and a reduction of compliances either by $10 \%$ (all aortic segments) or by $32 \%$ (all venous segments) [37].

The consequences of right kidney dysfunction on clinical measurables were simulated. The presence of right kidney dysfunction was programmed as either a large increase of right kidney resistance, or a large simultaneous reduction of aortic and right kidney compliances. Further, right kidney dysfunction was simulated as either homogeneous or inhomogeneous conditions. Under homogenous conditions, all microvascular resistances in the right kidney were either augmented 10 fold, or compliances reduced by $90 \%$, or both resistance and compliance alterations implemented simultaneously. Heterogenous right kidney dysfunction was simulated by manipulating three of the six right kidney microvascular beds' resistances (increased 10 fold) and compliances (reduced by $90 \%$ ). In addition, in accordance with experimental information [38,39], dysfunction of right kidney was also defined as either a 10 fold increase of the right kidney's resistance, a 90\% reduction of compliance (i.e., increase of stiffness) of the aorta (systemic artery), or both. Results using the latter definition of right kidney dysfunction are presented.

The HD sub-model simulates temporal changes of plasma volume under prescribed ultrafiltration targets. The efficacy of HD was assessed using the fraction change of plasma volume. The efficacy of HD was assessed under AF, exercise, and right kidney dysfunction conditions.

\subsection{Assessment of Parametric Sensitivity}

Sensitivity of the clinically relevant model outputs (see above) to model mechanism regulating parameters was computed using our implementation of partial ranked correlation coefficients [40]. The coefficients were used to rank the parameters in descending order of significance, and the most relevant results reported in this study.

\subsection{Numerical Methods}

The model was encoded into our C and MATLAB language based differential-algebraic equation solver suitable for efficient simulations [14]. The implicit solver is highly stable and provides high accuracy $\left(\mathrm{O}\left(\mathrm{dt}^{6}\right)\right)$ [41]. In all simulations, the maximum time step, $\mathrm{dt}$, was taken to be $0.005 \mathrm{~s}$ with tolerances (both, absolute and relative) of $10^{-6}$ for the adaptive time step solver. Whereas one instance of the model required a maximum of $180 \mathrm{~s}$ of serial run-time, a model population consisting of $10^{4}$ instances was generated within $3 \mathrm{~h}$ using 64 processors. The MATLAB code of the presented model is provided openly in our GitHub (https://github.com/mccsssk2/HumanCirculationModel2022_MDPI, accessed on 21 December 2021) repository. 


\section{Results}

\subsection{Baseline Model}

The baseline model population's aortic and kidney pressures as well as flows and shear are illustrated in Figure 3. The model's structure is provided in Figure 2 as well as Supplementary Materials, Figures S1-S3. Representative parameter Gaussian distributions used to generate the baseline population are shown in Supplementary Materials, Figure S4.
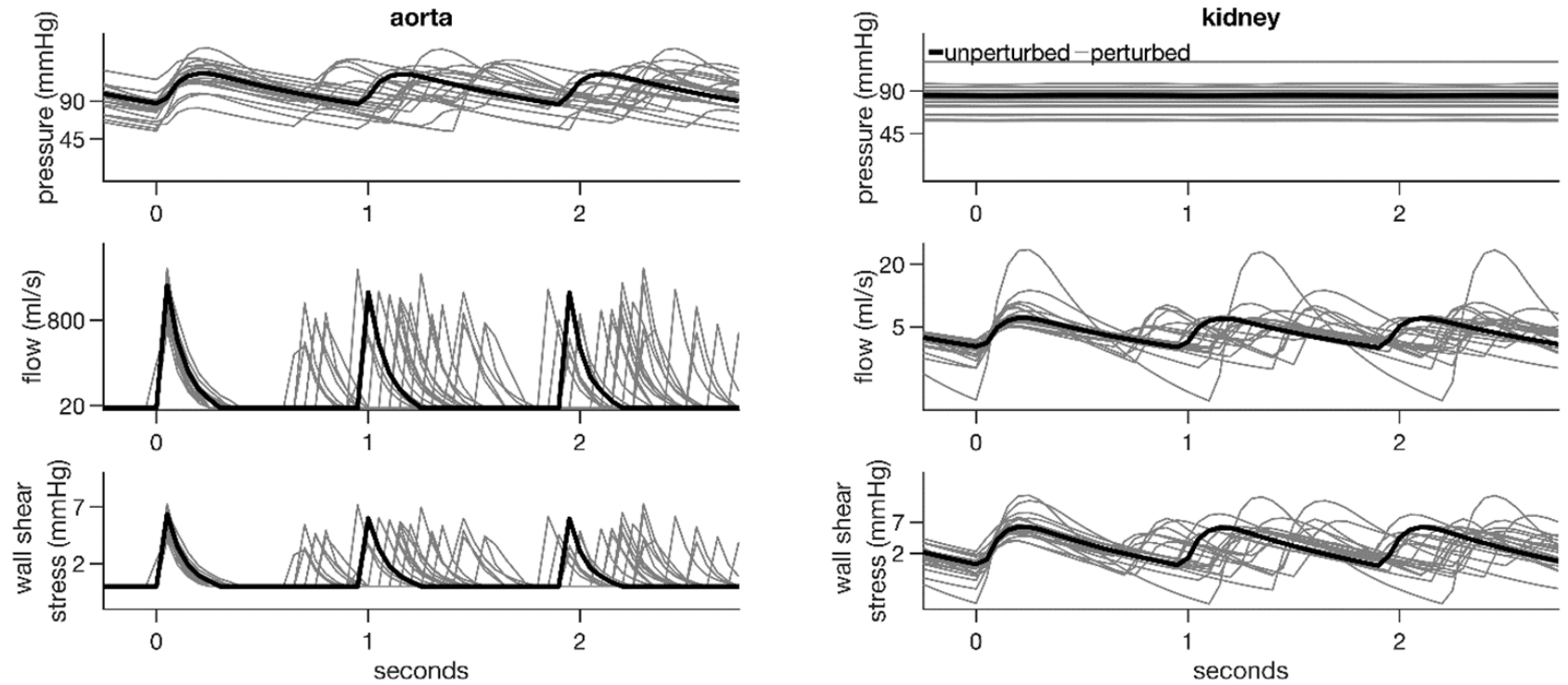

Figure 3. Representative profiles from the baseline simulated pressure (top row), blood flow (middle row), and wall shear stress (bottom row). Left column shows data for aorta and right column shows data for right kidney. In all panels, black lines show unperturbed simulation, while gray lines show instances from the population simulations obtained by perturbing parameters. See Section 2.2 for the method used to generate populations.

The model systemic artery's (aorta's) pressure has a diastolic value of $80 \mathrm{mmHg}$ and a systolic value of $120 \mathrm{mmHg}$ (Figure 3, top left). In contrast, the kidney inlet pressure was found to be constant at around $52 \mathrm{mmHg}$ (Figure 3, top right). Whereas the aortic blood flow was observed to vary between almost 0 to $1000 \mathrm{~mL} / \mathrm{s}$, the kidney's inlet flow oscillated between 4 and $6 \mathrm{~mL} / \mathrm{s}$ (Figure 3, second row). It was also observed that the kidney experienced beat to beat prolonged shear in comparison to the aorta (Figure 3, bottom row).

\subsection{Clinically Relevant Model Outputs}

The effects of HD, TH, and simultaneous HD with TH (HD\&TH) on clinically relevant measurables (outputs) are illustrated in Figure 4 . Whereas each bar represents the normalized mean relative to the displayed mean values, the black bar similarly represents the normalized standard deviation. TH led to a reduction in cardiac output (17\% under $\mathrm{TH}$, $14 \%$ under HD\&TH), while HD augmented it by a small amount ( $\%$ increase) (Figure $4 \mathrm{~A}$ ). While TH was found to augment heart rate, it was predominantly increased due to HD due to the underlying HD induced increase of intrinsic heart rate (Figure 4B, 20\% increase under HD). The diastolic and systolic pressures remained largely unaffected by TH, HD, or HD\&TH (Figure 4C,D). While shear stress (Figure 4E,F) was reduced by TH (15\% in the aorta, $6 \%$ in the kidney), HD augmented the same (5\% in both the aorta and kidney). Under HD\&TH conditions, shear was reduced in the aorta by $10 \%$ and in the right kidney by $3 \%$. Blood flow distributions in the four model populations (baseline, TH, HD, HD\&TH) are illustrated in Figure S5, while baroreflex control is shown in Figure S3. 

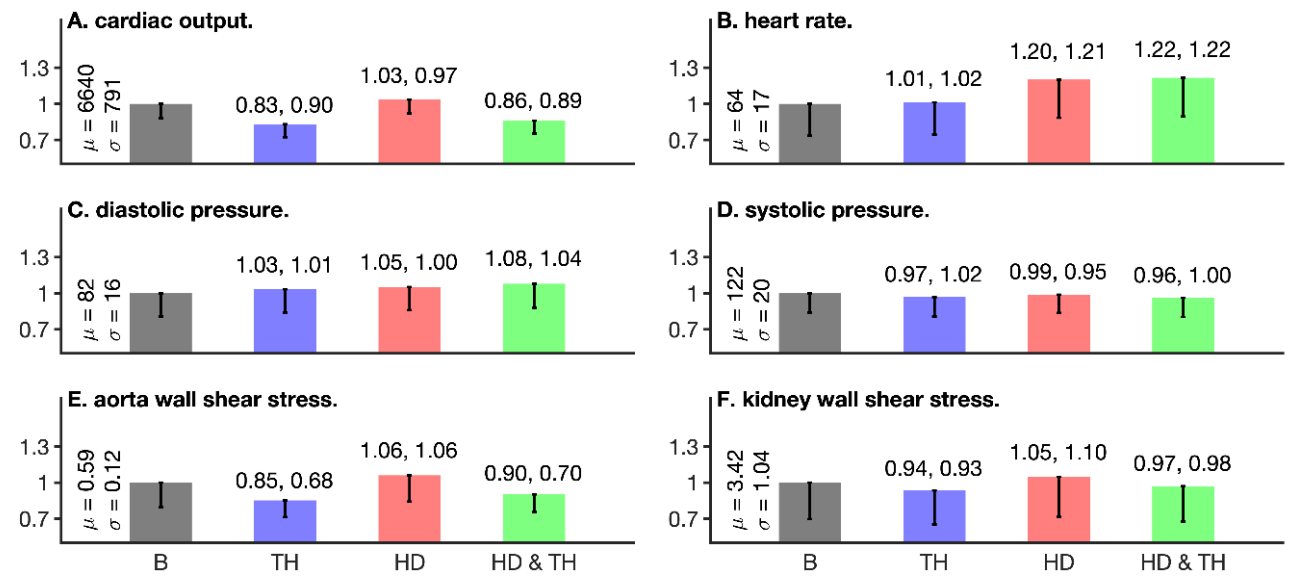

Figure 4. Model generated clinical measurables (model outputs) in the four populations (B, TH, HD, HD\&TH). In all panels, B (gray bars) represents baseline model outputs, TH (blue bars) represents model outputs under therapeutic hypothermia, HD (red bars) represents hemodialysis, and HD\&TH (green bars) represents simulation application of hemodialysis and therapeutic hypothermia. Panel (A) shows cardiac output, (B) shows heart rate, (C) shows diastolic cardiac pressure, (D) shows systolic cardiac pressure, (E) shows WSS in the aorta, and (F) shows wall shear stress in the right kidney. The black line in each bar represents the standard deviation. The mean values and standard deviations for baseline are provided in all panels. The mean values $(\mu)$ and standard deviations $(\sigma)$ relative to the respective baseline values are provided for TH, HD, and TH\&HD cases in all panels.

Whereas all outputs shown in Figure 4 are derived quantities based on the model's pressure and flow variables, their spread (represented by the standard deviations) was observed to be affected due to the underlying non-linear dynamical relationships. Overall, the spread of cardiac output and shear under TH and HD reduced, while the spread of heart rate increased. The increase in uncertainty in heart rate was observed to be predominantly due to HD (20\% increase of standard deviation, Figure 4B, red bar). All data in Sections 3.3 and 3.4 (Figures 5 and 6) are normalized to the respective baseline values of Figure 4 (gray bars).
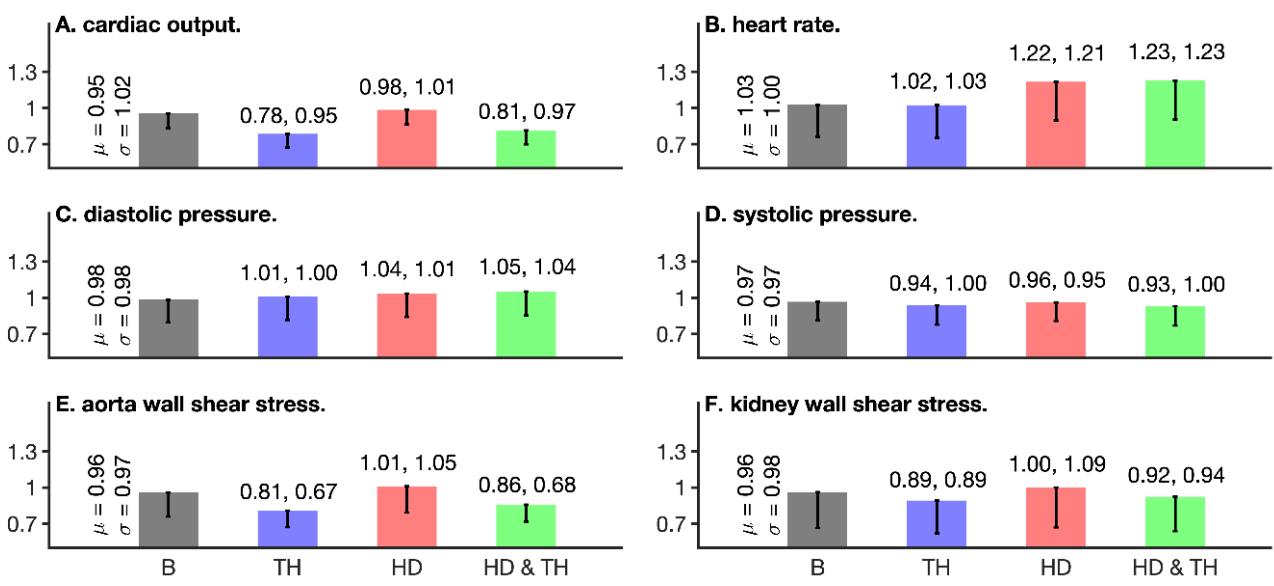

Figure 5. Effects of atrial fibrillation on clinical measurables. In all panels, data for atrial fibrillation [37] under baseline (B, gray bars), therapeutic hypothermia (TH, blue bars), dialysis (HD, red bars), and dialysis under TH (HD\&TH, green bars) conditions are shown. Panel (A) shows cardiac output, (B) shows heart rate, (C) shows diastolic cardiac pressure, (D) shows systolic cardiac pressure, (E) shows WSS in the aorta, and (F) shows wall shear stress in the right kidney. Mean values and standard deviations relative to the non-atrial fibrillation baseline case (Figure 3, gray bars and corresponding values) are provided in all panels. 

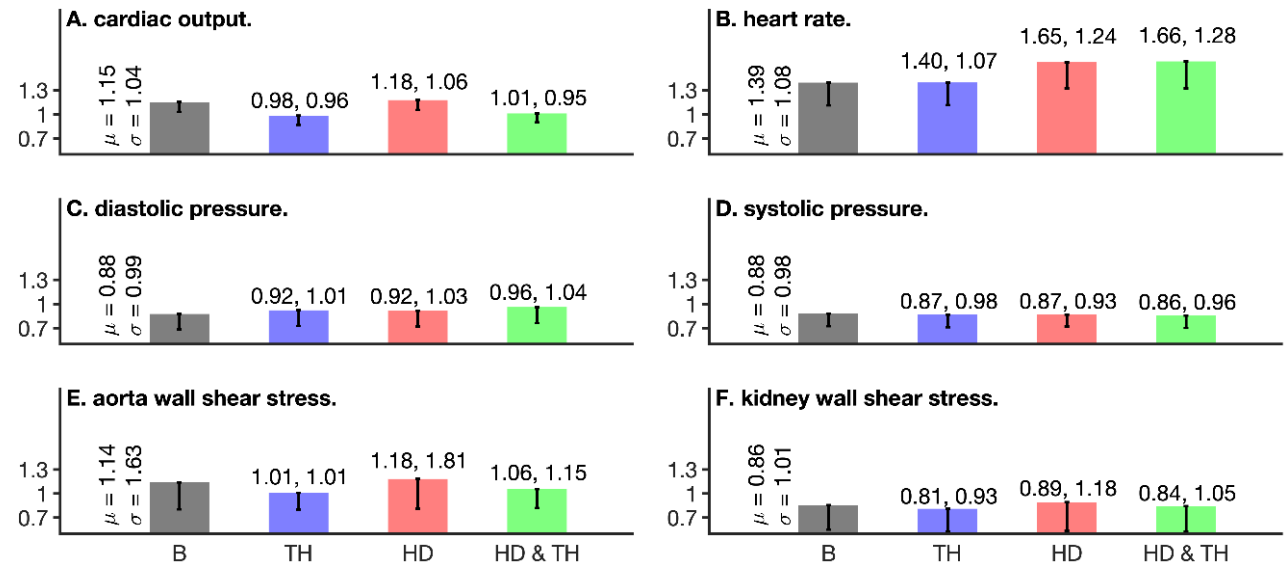

Figure 6. Effects of exercise on clinical measurables. In all panels, data for exercise [37] under baseline (B, gray bars), therapeutic hypothermia (TH, blue bars), dialysis (HD, red bars), and dialysis under TH (HD\&TH, green bars) conditions are shown. Panel (A) shows cardiac output, (B) shows heart rate, (C) shows diastolic cardiac pressure, (D) shows systolic cardiac pressure, (E) shows WSS in the aorta, and (F) shows WSS in the right kidney. Mean values and standard deviations relative to the non-exercise baseline case (Figure 3, gray bars and corresponding values) are provided in all panels.

\subsection{Role of Atrial Fibrillation}

AF conditions, as described in Section 2.4 Simulated conditions, were imposed on all four populations. The results (Figure 5) are presented in comparison to the non-AF case discussed in Section 3.2 and shown in Figure 4. Under baseline conditions (Figure 5, all gray bars), AF reduced cardiac output and blood pressure, increased heart rate, and had a heterogeneous effect on shear. In comparison to the non-AF case (Figure 4), AF reduced cardiac output in all four populations and the maximal reduction of $20 \%$ was under hypothermia (TH) conditions (Figure 5A, blue and green bars). HD has a small reducing effect on cardiac output (Figure 5A, red bar). The changes in spread of cardiac outputs in all AF affected populations was minimal in relation to the non-AF case (Figure 4). Under $\mathrm{AF}, \mathrm{HD}$ predominantly increased the heart rate by over $20 \%$ (Figure $5 \mathrm{~B}$, red and green bars) in relation to the non-AF baseline case (Figure 4B). Further, the spread of heart rates was maximally increased by HD under AF conditions (Figure 5B, error-bars of red and green bars). The diastolic and systolic blood pressures remained comparable to the respective baseline cases (Figure 4C,D, gray bars) under AF conditions (Figure 5C,D). AF conditions reduced shear in both the aorta (Figure 5E) and in the kidney (Figure 5F). The reduction was maximal under therapeutic hypothermia $(\mathrm{TH})$ conditions with a maximal reduction of $20 \%$ in the aorta and $11 \%$ in the kidney. Under HD\&TH conditions, the shear augmenting effects of TH were partially abrogated. In all cases (Figure 5E,F), the spread (intra-population standard deviation) of shear data was reduced by TH under AF conditions.

\subsection{Role of Exercise}

Exercise conditions were imposed on all four populations, and the model outputs (population means and standard deviations) compared to the non-exercise case of Figure 4 (Section 3.2). Under baseline conditions (Figure 6, gray bars), exercise increased cardiac output, increased heart rate, reduced blood pressures, and heterogeneously affected the shear. In comparison to the non-exercise case (Figure 4), exercise increased cardiac output by over $15 \%$ in the normothermic populations (Figure $6 \mathrm{~A}$, gray and red bars), and was unaffected by TH (Figure 6A, blue and green bars). The changes in spread of cardiac output in all exercise affected populations was minimal in relation to the non-exercise case (Figure 4). Under exercise, HD predominantly increased the heart rate by over $65 \%$ (Figure 6B, red and green bars). Heart rate was also increased under baseline-exercise and $\mathrm{TH}$-exercise conditions by $40 \%$ (Figure $6 \mathrm{~B}$, gray and blue bars). The population wide heterogeneity in heart rates was augmented due to HD (Figure 6B, red and green bars). 
The diastolic and systolic blood pressures were reduced by $8 \%$ in the baseline-exercise case (Figure 6C,D, gray bars). Both TH and HD conditions promoted higher diastolic but not systolic pressures (Figure 6C,D). The standard deviations of the population pressures remained unaffected in relation to the non-exercise case of Figure 4. Exercise conditions augmented shear in the aorta by $14 \%$ (Figure $4 \mathrm{E}$, gray bar) and reduced it in the kidney by $14 \%$ (Figure $6 \mathrm{~F}$, gray bar). Application of $\mathrm{TH}$ reduced shear in both the aorta (large vessel) and in the kidney (microvasculature) (Figure 6E,F, blue and green bars). HD was seen to be a shear augmenting factor under exercise. The effect of exercise in intrapopulation heterogeneity (standard deviation) was different among the large aorta and the smaller kidney vessels. In the aorta, the heterogeneity was augmented under baselineexercise and HD conditions, while the same was reduced in the kidney. TH reduced the shear heterogeneity.

\subsection{Role of One Homogenous Kidney Dysfunction}

Homogenous right kidney dysfunction was simulated by either augmenting all right renal microvascular resistances ten fold, or by reducing aortic compliance by $90 \%$ (Figure 7). Under non-failing right kidney conditions (Figure 7Ai,Aii), TH reduced inlet flow by 18\%. In contrast, HD augmented renal flow by $3 \%$ in each kidney. Under TH\&HD conditions, the renal flow was reduced by $14 \%$ (Figure $7 \mathrm{Ai}$,Aii, green bars) as compared to baseline (Figure 7Ai, gray bar). TH reduced the intra-population heterogeneity of renal flow (Figure 7Ai,Aii), while it was increased by HD.
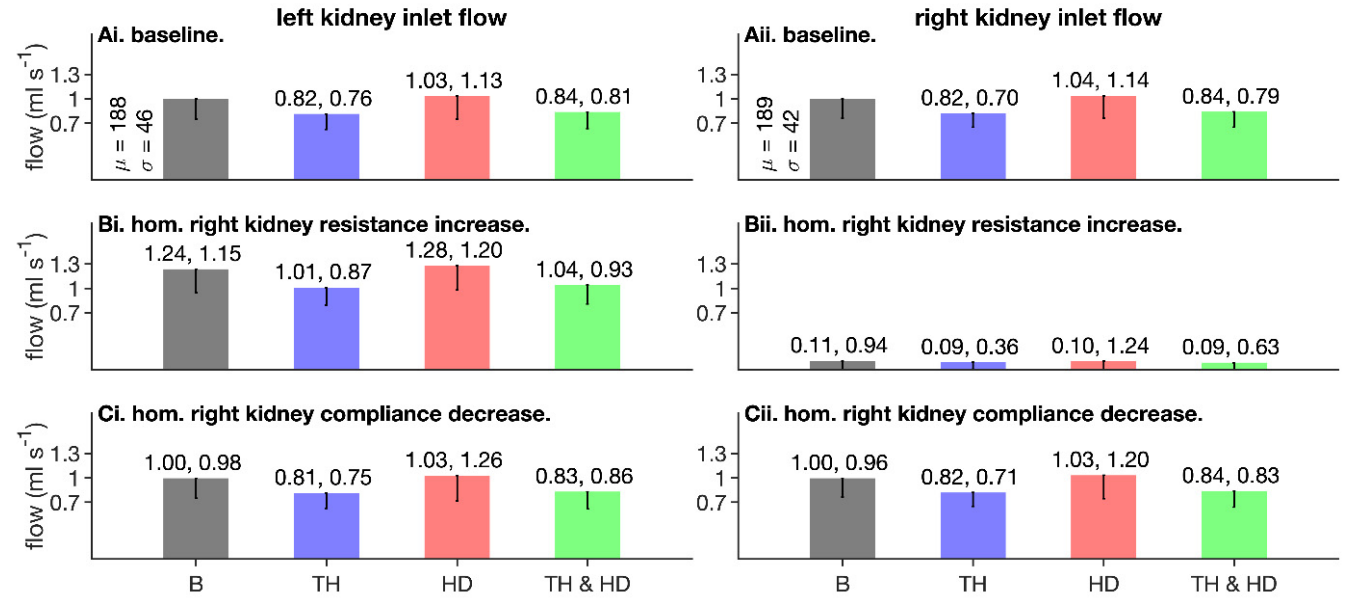

Figure 7. Effects of homogenous right kidney failure on left and right kidney inlet flow. Left column shows data for left kidney and right column shows data for right kidney. Top row shows data for control (absence of right kidney failure), middle row shows data when right kidney microvascular resistance was increased 10 fold, and bottom row shows data when right kidney microvascular compliance was reduced (i.e., stiffness was increased) by $90 \%$. In all panels, (B gray bars) represents baseline model outputs, TH (blue bars) represents model outputs under therapeutic hypothermia, HD (red bars) represents hemodialysis, and HD\&TH (green bars) represents simulation application of hemodialysis and therapeutic hypothermia. The mean values $(\mu)$ and standard deviations $(\sigma)$ for baseline (B, gray bars) are provided in panel $(\mathbf{A i})$. The mean values and standard deviations relative to the respective baseline values shown in (Ai) are provided for TH, HD, and TH\&HD cases in all panels.

When all microvascular resistances in the right kidney were increased by ten fold, the flow going to the right kidney virtually vanished (Figure 7Bii) and that to the left kidney increased (Figure 7Bi). The effects of TH, HD, and TH\&HD on left kidney flow under right renal failure (increased resistances) are shown in Figure 7Bi. In addition, TH reduced intrapopulation heterogeneity in both the left and right kidneys (reduced standard deviations), and it was augmented by HD.

Reduction of aortic and right kidney compliances did not affect inlet flow to either kidneys (Figure 7Ci,Cii, gray bars). Under TH conditions, both kidneys experienced reduced 
flow, while HD increased the flow by a small amount. TH\&HD conditions (Figure 7Ci,Cii, green bars) generated an $18 \%$ reduction of flow to both kidneys. While HD increased intra-population heterogeneity in flow by $22 \%$ due to increased stiffness, $\mathrm{TH}$ was found to reduce it by almost $30 \%$. Under TH\&HD conditions, the heterogeneity was seen to be reduced in comparison to the respective control value of Figure 7Ai, gray bar.

\subsection{Role of Heterogeneous Right Kidney Dysfunction}

Populations where half ( 3 of the six renal microvascular beds in the right kidney) of the right renal microvasculature was increased by 10 fold or compliances reduced by $90 \%$ to represent heterogenous right kidney dysfunction. Similar to the homogeneous case (Section 3.5, above), TH reduced renal inlet flow, while HD augmented the same. Heterogeneous right renal dysfunction represented by resistance increase was unable to affect renal inlet flows as much as the corresponding homogeneous case (Figure 8Bi,Bii). Altered compliances had a marginal effect on renal inlet (Figure 8Ci,Cii).
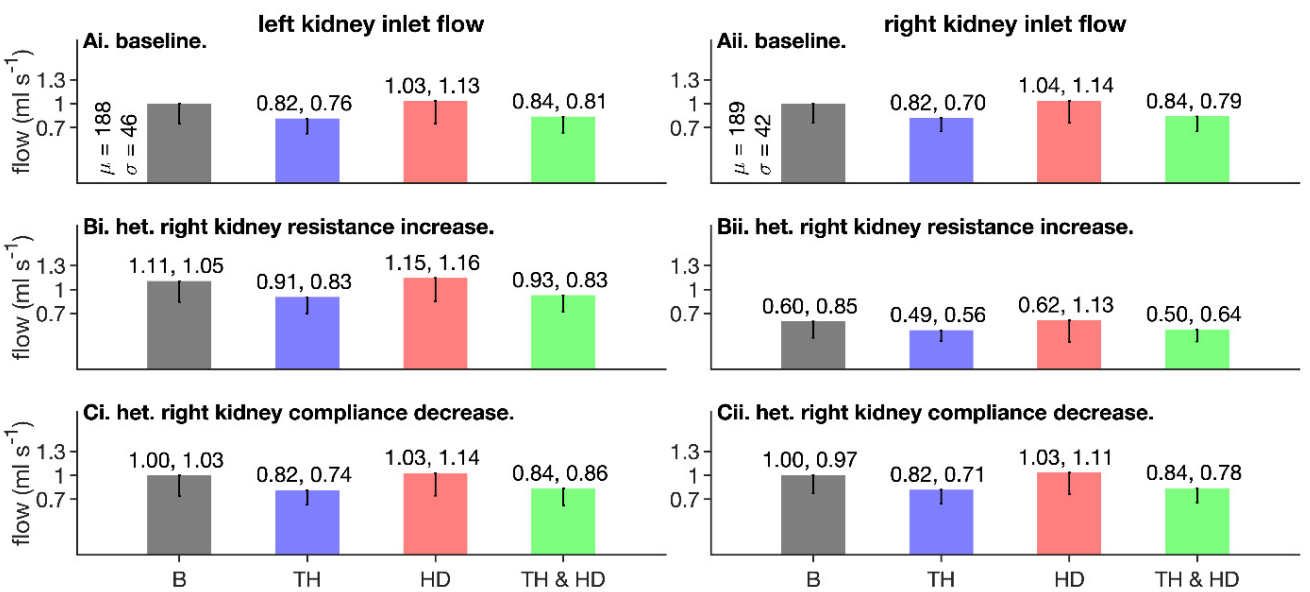

Figure 8. Effects of heterogeneous right kidney failure on renal inlet flow. Left column shows data for left kidney and right column shows data for right kidney. Top row shows data for control (absence of right kidney failure), middle row shows data when half of the right kidney microvascular resistances were increased 10 fold, and bottom row shows data when half of the right kidney microvascular compliances were reduced (i.e., stiffness was increased) by $90 \%$. See Supplementary Materials, Figure S1 for the model's kidney structure. In all panels, (B gray bars) represents baseline model outputs, TH (blue bars) represents model outputs under therapeutic hypothermia, HD (red bars) represents hemodialysis, and HD\&TH (green bars) represents simulation application of hemodialysis and therapeutic hypothermia. The mean values $(\mu)$ and standard deviations $(\sigma)$ for baseline (B, gray bars) are provided in panel (Ai). The mean values and standard deviations relative to the respective baseline values shown in (Ai) are provided for TH, HD, and TH\&HD cases in all panels.

The more significant role of microvascular resistance over compliance is illustrated in Figure 9. At each value of compliance and resistance, the six flows in each kidney were noted, and their heterogeneity representing standard deviation used to construct the result (Figure 9). As is evident, resistance readily promoted heterogeneity in the affected kidney (right kidney), while compliance plays a marginal role in the presented model. 

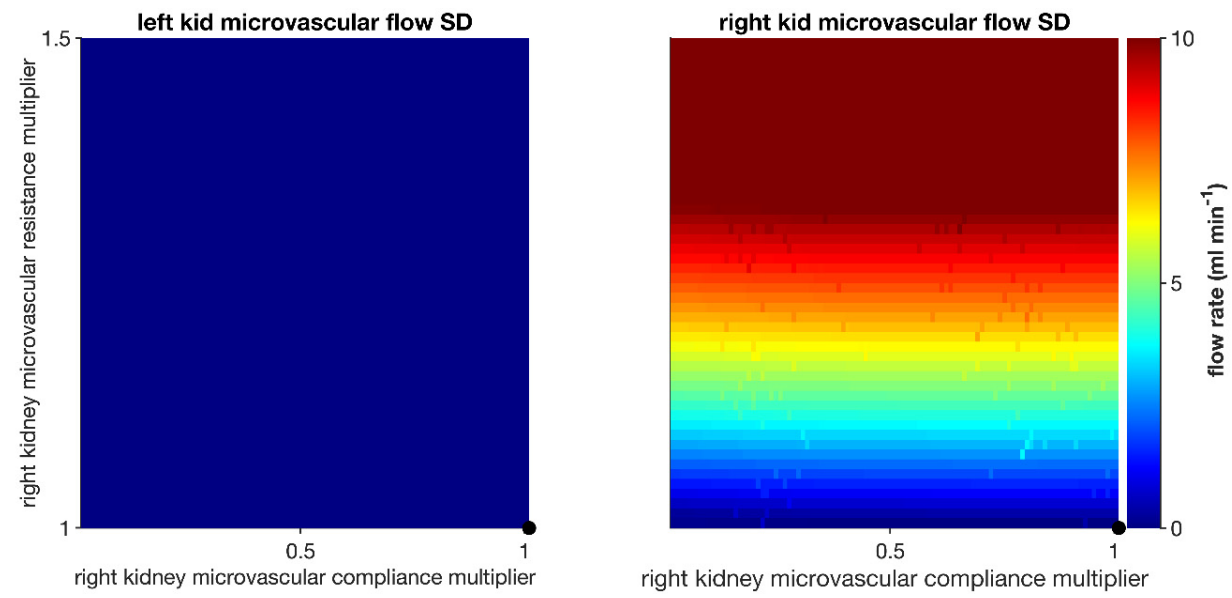

Figure 9. Mean standard deviation of blood flow in renal microvasculature representing blood flow heterogeneity. The left kidney (left panel) was seen to receive uniform blood flow, while the right kidney's (right panel) microvascular blood flow was seen to be heterogeneous. See Section 2.4 for details, and results section for an expanded description of the result.

\subsection{Effectiveness of Volume Removal under Diseased Conditions}

Plasma volume removal under HD was assessed by the relative change of plasma volume before and after a HD session. Under normothermia and standard target ultrafiltrate (Figure 10Ai), atrial fibrillation, exercise, as well as right renal failure reduced HD efficacy, as reflected in the reduced changes to plasma volume. The effect of exercise was maximal where HD removed $40 \%$ less volume as compared to the baseline case (Figure 10Ai, purple and gray bars respectively). TH did not affect the plasma volume removal (Figure 10Aii). Under low target UF (Figure 10Bi,Bii), the alteration of plasma volume was higher and under high target UF (Figure 10Ci,Cii) the alteration of plasma volume was lower. TH was not seen to have any role in plasma volume removal efficacy in the presented model. In comparison to control (Figure 10Ai, gray bar), all conditions reduced the intra-population heterogeneity at all target UF values. Further, TH further reduced the heterogeneity of HD efficacy (Figure 10, right column).
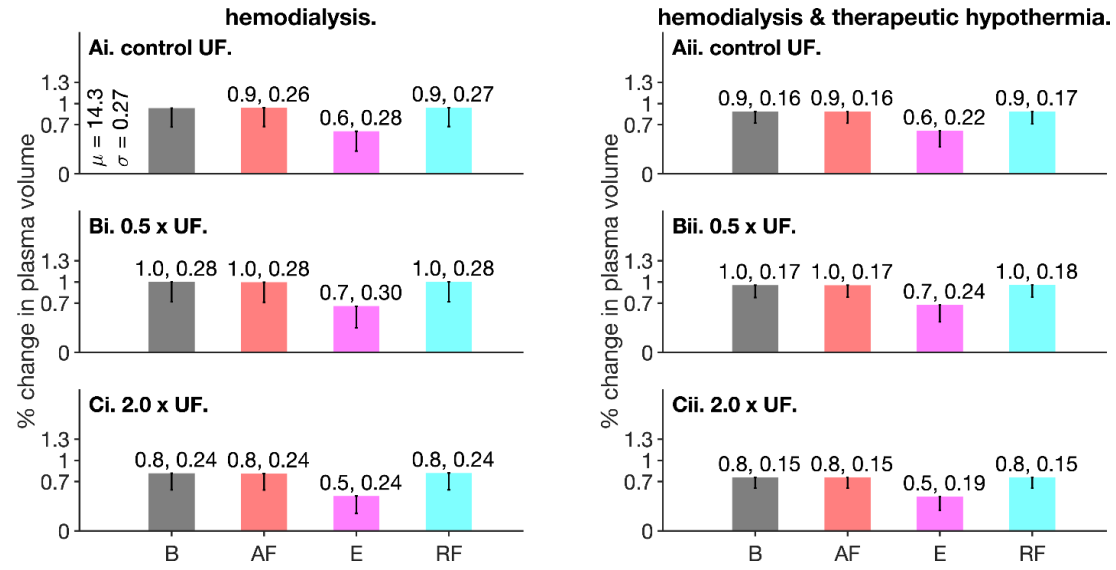

Figure 10. Effectiveness of water volume removal due to HD under multiple conditions and target ultrafiltrate (UF). Left column shows data under hemodialysis conditions, and right column shows data under hemodialysis with therapeutic hypothermia conditions. In all panels, (B gray bars) represents the baseline population, AF (red bars) represents the atrial fibrillation population, $\mathrm{E}$ (purple bars) represents the exercise population, and RF (cyan bars) represents the right kidney failure population. All values and standard deviations were normalized to the respective baseline means $(\mu)$ and standard deviations $(\sigma)$ which are shown besides the gray bar in panel (Ai). 


\subsection{Most Significant Model Parameters}

The results of PRCC based sensitivity analysis are illustrated in Figure 11. Cardiac output (Figure 11A) is positively modulated by the intrinsic heart rate and systolic elastance of the right ventricle under all four conditions (baseline, HD, TH, and HD\&TH), while it is negatively modulated by the diastolic right ventricle elastance, upper body resistance, and the baroreflex mechanism parameter, G. Additionally, HD, TH, as well as TH\&HD markedly alter the sensitivity of cardiac output to the right ventricle elastance as well as the baroreflex control mechanism. The heart rate (Figure 11B) is maximally modulated by the intrinsic heart rate and the baroreflex mechanism, while resistances (splanchnic and lower body) and right ventricle diastolic elastance play a secondary but significant role. While heart rate depends positively on the intrinsic heart rate and diastolic right ventricle elastance, it is modulated negatively by the baroreflex and resistance mechanisms. The systemic artery's systolic (Figure 11C) as well as diastolic (Figure 11D) pressures are maximally modulated by the baroreflex. Resistances and elastances play an important role in modulating both. Further, the systemic artery's compliance (aortic compliance) negatively modulates the systolic pressure while it positively modulates diastolic pressure (Figure 11C,D). Blood vessel shear was seen to be modulated by intrinsic heart rate, baroreflex, elastances, and microvascular resistances. The ranked PRCC sensitivities to shear are shown in Figure 11E,F.
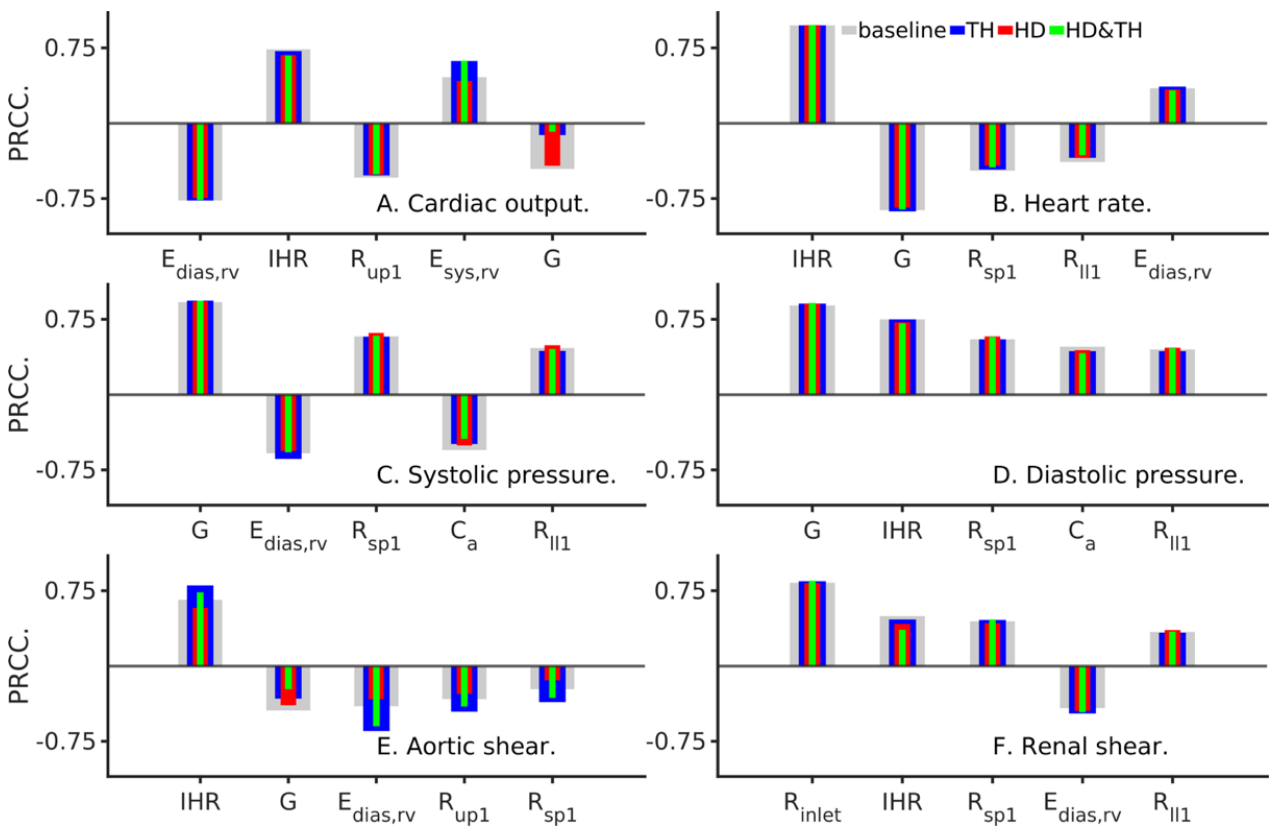

Figure 11. Sensitivity of clinically relevant model outputs to parameters. In all panels, $\mathrm{E}_{\mathrm{dias}, \mathrm{rv}}$ represents diastolic right ventricle elastance; $E_{\text {sys,rv }}$ represents systolic right ventricle elastance; IHR represents intrinsic heart rate; $R_{\text {up } 1}$ represents upper body resistance; $G$ represents baroreflex regulatory parameter; $R_{s p 1}$ represents splanchnic resistance; $R_{111}$ represents legs resistance; $C_{a}$ represents aorta compliance; $R_{\text {inlet }}$ represents kidney inlet resistance.

\section{Conclusions and Discussion}

A model that combined whole body circulation with detailed kidneys representation, along with baroreflex control and dialysis unit was developed. While the present model derives from previous developments by others [21,30,31,42], this work used it in assessing a novel therapy (therapeutic hypothermia) pertinent to our clinical research. As seen in Figure 3, our implementation is capable of qualitatively reproducing flow-pressure waveforms [43], in addition to providing difficult to measure information such as shear as well as population wide expectation. 
In line with our clinical observations, the model suggests that HD augments cardiac output, heart rate, as well as shear stress (Figure 4). While HD induced augmentation of heart rates and shears may be undesirable side effects [44], TH appears to impart benefit by reducing heart rates and normalizing vascular shear stress, in agreement with clinical finding [45]. It can be appreciated that the normalized wall shear stress is a direct result of vascular diameter reduction (Equation (1)) leading to an augmented microvascular increase in resistance while cardiac output reduces to a certain extent.

Atrial fibrillation (AF) is a prevalent condition among critically ill patients such as those with renal failure. The presented model suggests that AF potentially exacerbates the HD induced augmentation of heart rate (Figure 5B) and lowers blood pressure (Figure 5C,D). Therapeutic hypothermia neither reduced the HD induced heart rate increase, nor did it reverse the HD induced lowered blood pressure. However, the model does suggest that shear stress in blood vessels, due to its lower intra-population heterogeneity, is strongly linked to underlying AF. The simulated AF had a marginal effect on model behaviour, an observation that is aligned with the findings of Anselmino et al. [37]. The benefit of TH under AF conditions appears to be reduction of both shear stress and intra-population heterogeneity.

The modelled exercise conditions (Figure 6) increased cardiac outputs but beneficially lowered systolic blood pressure. TH imparts further benefit under exercise by primarily in reducing large vessel (aortic) as well as small vessel (renal) shear, which may provide hemodynamic stability.

Within the confines of the model, renal resistance appears to affect renal flow more than renal compliance (Figures 7 and 8). The present findings are aligned with our previous findings [14] where large vessel compliance and small vessel resistance was found to affect overall whole body hemodynamics. Our recent clinical observations in renal failure patients show that dialysis increased blood flow heterogeneity in visceral organs, while therapeutic hypothermia may reduce it. Figures 7 and 8 show that the cause may be found within the population wide heterogeneity measure of standard deviation, or in the absolute measurement of microvascular flow heterogeneity in the organ (Figure 9).

The effectiveness of dialysis was assessed using relative changes of plasma volume (Figure 10). As a simple definition, a larger fraction of plasma volume removed from the body by the dialyzer was defined as more effective HD. TH reduced the amount of plasma volume removed. In addition, pathological alterations of the hemodynamics such as atrial fibrillation and renal failure were also seen to reduce the amount of plasma volume to be removed by dialysis. Surprisingly, exercise caused the minimal amount of plasma volume to be removed.

Sensitivity analysis, using partial ranked correlation coefficients, permitted ranking of model parameters. Among the large number of model parameters, a handful emerged to be the most relevant (Figure 11). Identifying the parametric regulation of outputs will permit future development of the model mechanisms. As expected, the baroreflex control (represented by parameter $\mathrm{G}$ ) regulates multiple model outputs of interest. Furthermore, the sensitivity of the model to baroreflex control appears to be reduced significantly by TH. The sensitivity of blood vessel shear also appears to be affected by the right ventricle elastance, as well as peripheral resistances (splanchnic and upper body).

Limitations: The present model is primed for further development. Implementation of detailed organ level vasculature representations of cerebral [46], hepatic [47], pulmonaryrespiratory [48], cardio-pulmonary [49], and other will better complement future clinicalmodelling studies. Future inclusion of myogenic hemodynamic regulation $[35,50]$ will extend the spectrum of phenomena explored [51] and further refine the presented results. In addition to baroreflex control, vascular tone is known to be significantly regulated by renal function. The inclusion of biochemical mechanisms of gas exchange and metabolism [52,53] will extend the model to permit drug testing, in addition to the presently tested physical therapy of TH. The inclusion of biochemical mechanisms is particularly relevant as we will extend our descriptions of organs as well as the dialyzer as an artificial kidney. The model will be extended to simulate ionic regulation of vascular tone [54-56]. Whereas 
the presented dialyzer affects blood composition, the known effect that the dialyzer also affects the circulation [57] remains to be incorporated into the model. Complementary to phenomenological hemodynamics, a significant proportion of mechanisms at tissue and cellular levels are expected to be valuable in our future modelling-clinical-imaging approach. We are currently constructing multiscale cell to vasculature models driven by current understanding (e.g., see [54]) that will assist the dissection of pathophysiological disease mechanisms and pharmacological targets. The mechanisms and pathways of widespread sepsis infections during HD could be further illuminated using the multi-scale models [58].

Whereas the presented model incorporates parametric information representing specific patients (either dialyzed, hypothermic, atrial fibrillation, or exercised), the simulations may generate generic than specific populations as experimental data was unavailable to the study. In depth disease specific parameter identification using data along with improved population generating methods $[59,60]$ may provide better insights in our future studies. Doing so will inevitably permit uncovering of mechanisms and assist to make novel predictions.

Often, the simulation of patient populations is driven by known parameter variability as well as inter-parameter independence $[59,61,62]$. While neither have been considered in this study, each of our populations encompasses a wide range of parameters representing inter-patient variability.

Due to model limitations, the model may generate somewhat unrealistic and qualitative results. However, we believe that our results will largely remain valid in light of future model development.

Summary of conclusions: To summarize, while $\mathrm{HD}, \mathrm{AF}$, and renal failure appear to be deleterious, TH and exercise may provide some benefit to the patient (Figure 1). We found that HD and AF give rise to undesirable augmentation of wall shear stress which may increase vascular inflammation. TH and exercise treatments may reduce the inflammation by reducing wall shear stress, and may also improve HD efficacy. However, microvascular disease such as renal failure may blunt the effectiveness of $\mathrm{TH}$ and exercise treatments.

\section{Data Availability}

The model source code is made openly available via GitHub using which all data in this work can be reproduced.

Supplementary Materials: The following supporting information can be downloaded at: https: / / www.mdpi.com/article/10.3390/app12010307/s1, Table S1: Model resistance, compliance, and cardiac elastance parameters used to generate populations; Figure S1: Schematic diagram of the detailed right-left kidneys' complex. Each kidney consists of six microvascular components, implemented as three element Windkessel models. Each component consists of two microvascular beds that is assigned two resistance values and one compliance value. All resistances and com-pliances under baseline conditions were assumed to have the same value (see Table S1 for pa-rameter values); Figure S2: Schematic of the dialysis unit adapted from the literature. Further details including baseline parameter values used in this work; Figure S3: Schematic of the baroreflex sub-model; Figure S4: Representative parameter Gaussian distributions in the baseline model population (see Figure 2, main manuscript) where mean values are taken from the unperturbed model, and a coefficient of variation of 0.25 is used to generate the population. Left panel shows distribution of aortic compliance, and right panel shows distribution of left kidney inlet resistance; Figure S5: Distribution of flows in model organs under baseline (grey), hemodialysis (red), therapeutic hypothermia (blue), and simultaneous hemodialysis with therapeutic hypothermia (green). Top row: upper body; second row: splanchnic; third row: lower body; bottom row: two kidney com-plex. This illustration accompanies Figures 2 and 3 in the main manuscript; Figure S6: Baroreflex control of heart rate by the parasympathetic and sympathetic tone mechanisms. The control value is shown by the black circle in the bottom left hand corner of the panel. Both parasympathetic (horizontal axis) and sympathetic (vertical axis) rate values are shown after subtracting the base-line value. See Table S1 for parameter values; Figure S7: Blood flow under baseline (left column), AF (middle column), and exercise (right 
column) conditions. All data are normalized to the baseline values of the left panel; Figure S8: Population distributions of intracellular volume (left panel), interstitial volume (middle panel), and plasma volume (right panel). See Figures 2 and 3 and related text for details.

Author Contributions: Study design: J.J.J., S.R.K., D.G. and C.W.M.; model development, J.J.J., T.J.H., S.R.K. and C.S. simulations: J.J.J. and S.R.K.; data analysis and figures, J.J.J., T.J.H. and C.S.; draft writing, J.J.J., C.S. and S.R.K.; draft completion, S.R.K.; final manuscript, S.R.K., J.J.J., D.G. and C.W.M.; funding secured by, S.R.K., C.W.M. and D.G. All authors have read and agreed to the published version of the manuscript.

Funding: This research was funded by Canada Canarie Inc. grant number [RS3-111], Canada Heart and Stroke Foundation grant number [G-20-0028717] and the APC was funded by the publishing MDPI journal.

Institutional Review Board Statement: This study did not use sensitive data, patient information, or any other identity data. This computational study did not require ethics approvals of any nature.

Informed Consent Statement: This computational study did not require consent from patients or otherwise.

Data Availability Statement: The MATLAB source code to reproduce all data in this work is openly available in our lab's github repository with URL: https:/ / github.com/mccsssk2/HumanCirculationModel202 2_MDPI (accessed on 21 December 2021). Computationally efficient source code can be obtained from the corresponding authors upon reasonable request.

Acknowledgments: This work was supported by Canada Canarie Inc. (project: RS3-111), partly by Canadian Heart and Stroke Foundation grant (G-20-0028717, PI: CWM), and partly by Canadian NSERC grant (R4081A03, PI: Daniel Goldman). This work is part of CS's research based undergraduate training in Western University. SRK also thanks Lawson Health Research Institute, Western University, MITACS Globalink (Canada), and Compute Canada for funding, compute, labour, and other resources.

Conflicts of Interest: The authors declare no conflict of interest.

\section{References}

1. Hill, N.R.; Fatoba, S.T.; Oke, J.L.; Hirst, J.A.; O'Callaghan, C.A.; Lasserson, D.S.; Hobbs, F.D. Global Prevalence of Chronic Kidney Disease-A Systematic Review and Meta-Analysis. PLOS ONE 2016, 11, e0158765. [CrossRef]

2. McIntyre, C.W. Recurrent circulatory stress: The dark side of dialysis. Semin. Dial. 2010, 23, 449-451. [CrossRef] [PubMed]

3. Burton, J.O.; Jefferies, H.J.; Selby, N.M.; McIntyre, C.W. Hemodialysis-induced cardiac injury: Determinants and associated outcomes. Clin. J. Am. Soc. Nephrol. CJASN 2009, 4, 914-920. [CrossRef] [PubMed]

4. Marants, R.; Qirjazi, E.; Grant, C.J.; Lee, T.Y.; McIntyre, C.W. Renal Perfusion during Hemodialysis: Intradialytic Blood Flow Decline and Effects of Dialysate Cooling. J. Am. Soc. Nephrol. JASN 2019, 30, 1086-1095. [CrossRef] [PubMed]

5. Qirjazi, E.; Salerno, F.R.; Akbari, A.; Hur, L.; Penny, J.; Scholl, T.; McIntyre, C.W. Tissue sodium concentrations in chronic kidney disease and dialysis patients by lower leg sodium-23 magnetic resonance imaging. Nephrol. Dial. Transplant. 2020, 7, 1234-1243. [CrossRef]

6. Kharche, S.R.; So, A.; Salerno, F.; Lee, T.Y.; Ellis, C.; Goldman, D.; McIntyre, C.W. Computational Assessment of Blood Flow Heterogeneity in Peritoneal Dialysis Patients' Cardiac Ventricles. Front. Physiol. 2018, 9, 511. [CrossRef]

7. Burton, J.; Selby, N.; Leccisotti, L.; Korsheed, S.; McIntyre, C.; Camici, P. Haemodialysis induced contractile dysfunction is associated with acute and reversible reduction in global and segmental myocardial blood flow. Circulation 2007, $116,757$. [CrossRef]

8. Manns, B.J.; Mendelssohn, D.C.; Taub, K.J. The economics of end-stage renal disease care in Canada: Incentives and impact on delivery of care. Int. J. Health Care Financ. Econ. 2007, 7, 149-169. [CrossRef]

9. Al-Jaishi, A.A.; McIntyre, C.W.; Sontrop, J.M.; Dixon, S.N.; Anderson, S.; Bagga, A.; Benjamin, D.; Berry, D.; Blake, P.G.; Chambers, L.; et al. Major Outcomes With Personalized Dialysate TEMPerature (MyTEMP): Rationale and Design of a Pragmatic, Registry-Based, Cluster Randomized Controlled Trial. Can. J. Kidney Health Dis. 2020, 7, 2054358119887988. [CrossRef]

10. Yeung, K.K.; De Gouyon Matignon, C.; Renwarin, L.; Tjon, A.F.M.R.; Teerlink, T.; van Leeuwen, P.A.; Musters, R.J.; Wisselink, W.; Tangelder, G.J. Hypothermic renal perfusion during aortic surgery reduces the presence of lipocalin-2 and preserves renal extraction of dimethylarginines in rats. Am. J. Physiol. Ren. Physiol. 2011, 301, F1231-F1241. [CrossRef]

11. Shang, J.K.; Esmaily, M.; Verma, A.; Reinhartz, O.; Figliola, R.S.; Hsia, T.Y.; Feinstein, J.A.; Marsden, A.L. Patient-Specific Multiscale Modeling of the Assisted Bidirectional Glenn. Ann. Thorac. Surg. 2019, 107, 1232-1239. [CrossRef]

12. Shi, Y.; Korakianitis, T.; Bowles, C. Numerical simulation of cardiovascular dynamics with different types of VAD assistance. J. Biomech. 2007, 40, 2919-2933. [CrossRef] [PubMed] 
13. Shi, Y.; Lawford, P.; Hose, R. Review of zero-D and 1-D models of blood flow in the cardiovascular system. Biomed. Eng. Online 2011, 10, 33. [CrossRef]

14. Altamirano-Diaz, L.; Kassay, A.D.; Serajelahi, B.; McIntyre, C.W.; Filler, G.; Kharche, S.R. Arterial Hypertension and Unusual Ascending Aortic Dilatation in a Neonate With Acute Kidney Injury: Mechanistic Computer Modeling. Front. Physiol. 2019, 10, 1391. [CrossRef]

15. Heldt, T.; Shim, E.B.; Kamm, R.D.; Mark, R.G. Computational modeling of cardiovascular response to orthostatic stress. J. Appl. Physiol. 2002, 92, 1239-1254. [CrossRef]

16. Heldt, T.; Mukkamala, R.; Moody, G.B.; Mark, R.G. CVSim: An Open-Source Cardiovascular Simulator for Teaching and Research Open Pacing Electrophysiol. Ther. J. 2010, 3, 45-54.

17. Diaz-Artiles, A.; Heldt, T.; Young, L.R. Computational model of cardiovascular response to centrifugation and lower body cycling exercise. J. Appl. Physiol. 2019, 127, 1453-1468. [CrossRef]

18. Pallone, T.L.; Hyver, S.; Petersen, J. The simulation of continuous arteriovenous hemodialysis with a mathematical model. Kidney Int. 1989, 35, 125-133. [CrossRef]

19. Annan, K. Mathematical modeling of the dynamic exchange of solutes during bicarbonate dialysis. Math. Comput. Model. 2012, 55, 1691-1704. [CrossRef]

20. Aniort, J.; Chupin, L.; Cîndea, N. Mathematical model of calcium exchange during haemodialysis using a citrate containing dialysate. Math. Med. Biol. J. IMA 2017, 35, I87-I120. [CrossRef] [PubMed]

21. Coli, L.; Ursino, M.; Dalmastri, V.; Volpe, F.; La Manna, G.; Avanzolini, G.; Stefoni, S.; Bonomini, V. A simple mathematical model applied to selection of the sodium profile during profiled haemodialysis. Nephrol Dial. Transplant. 1998, 13, 404-416. [CrossRef]

22. Pietribiasi, M.; Waniewski, J.; Wojcik-Zaluska, A.; Zaluska, W.; Lindholm, B. Model of fluid and solute shifts during hemodialysis with active transport of sodium and potassium. PLOS ONE 2018, 13, e0209553. [CrossRef] [PubMed]

23. Pietribiasi, M.; Waniewski, J.; Zaluska, A.; Zaluska, W.; Lindholm, B. Modelling Transcapillary Transport of Fluid and Proteins in Hemodialysis Patients. PLoS ONE 2016, 11, e0159748. [CrossRef] [PubMed]

24. Werynski, A.; Waniewski, J. Theoretical description of mass transport in medical membrane devices. Artif. Organs 1995, 19, 420-427. [CrossRef]

25. Maheshwari, V.; Thijssen, S.; Tao, X.; Fuertinger, D.; Kappel, F.; Kotanko, P. A novel mathematical model of protein-bound uremic toxin kinetics during hemodialysis. Sci. Rep. 2017, 7, 10371. [CrossRef] [PubMed]

26. Ursino, M.; Coli, L.; Brighenti, C.; Chiari, L.; de Pascalis, A.; Avanzolini, G. Prediction of solute kinetics, acid-base status, and blood volume changes during profiled hemodialysis. Ann. Biomed. Eng. 2000, 28, 204-216. [CrossRef] [PubMed]

27. Lin, C.L.; Tawhai, M.H.; Hoffman, E.A. Multiscale image-based modeling and simulation of gas flow and particle transport in the human lungs. Wiley Interdiscip. Rev. Syst. Biol. Med. 2013, 5, 643-655. [CrossRef]

28. deBoer, R.W.; Karemaker, J.M.; Strackee, J. Hemodynamic fluctuations and baroreflex sensitivity in humans: A beat-to-beat model. Am. J. Physiol. 1987, 253, H680-H689. [CrossRef] [PubMed]

29. Schuessler, R.B.; Canavan, T.E.; Boineau, J.P.; Cox, J.L. Baroreflex modulation of heart rate and initiation of atrial activation in dogs. Am. J. Physiol. 1988, 255, H503-H513. [CrossRef]

30. Lim, K.M.; Choi, S.W.; Min, B.G.; Shim, E.B. Numerical Simulation of the Effect of Sodium Profile on Cardiovascular Response to Hemodialysis. Yonsei Med. J. 2008, 49, 581-591. [CrossRef]

31. Lin, J.; Ngwompo, R.F.; Tilley, D.G. Development of a cardiopulmonary mathematical model incorporating a baro-chemoreceptor reflex control system. Proc. Inst. Mech. Eng. Part H J. Eng. Med. 2012, 226, 787-803. [CrossRef]

32. Fernandez, J.F.; Criado, C. Algorithm for normal random numbers. Phys. Rev. E Stat. Phys. Plasmas Fluids Relat. Interdiscip. Top. 1999, 60, 3361-3365. [CrossRef]

33. Calvo, M.; Rolle, V.L.; Romero, D.; Behar, N.; Gomis, P.; Mabo, P.; Hernandez, A.I. Global Sensitivity Analysis of a Cardiovascular Model for the Study of the Autonomic Response to Head-up Tilt Testing. In Proceedings of the 40th Annual International Conference of the IEEE Engineering in Medicine and Biology Society, Honolulu, HI, USA, 17-21 July 2018 ; pp. 5458-5461.

34. Konstas, A.A.; Neimark, M.A.; Laine, A.F.; Pile-Spellman, J. A theoretical model of selective cooling using intracarotid cold saline infusion in the human brain. J. Appl. Physiol. 2007, 102, 1329-1340. [CrossRef]

35. Arciero, J.C.; Carlson, B.E.; Secomb, T.W. Theoretical model of metabolic blood flow regulation: Roles of ATP release by red blood cells and conducted responses. Am. J. Physiol. Heart Circ. Physiol. 2008, 295, H1562-H1571. [CrossRef]

36. Huo, Y.; Linares, C.O.; Kassab, G.S. Capillary perfusion and wall shear stress are restored in the coronary circulation of hypertrophic right ventricle. Circ. Res. 2007, 100, 273-283. [CrossRef]

37. Anselmino, M.; Scarsoglio, S.; Saglietto, A.; Gaita, F.; Ridolfi, L. A Computational Study on the Relation between Resting Heart Rate and Atrial Fibrillation Hemodynamics under Exercise. PLoS ONE 2017, 12, e0169967. [CrossRef]

38. Amann, K.; Wolf, B.; Nichols, C.; Tornig, J.; Schwarz, U.; Zeier, M.; Mall, G.; Ritz, E. Aortic changes in experimental renal failure: Hyperplasia or hypertrophy of smooth muscle cells? Hypertension 1997, 29, 770-775. [CrossRef]

39. Lin, C.Y.; Hsu, S.C.; Lee, H.S.; Lin, S.H.; Tsai, C.S.; Huang, S.M.; Shih, C.C.; Hsu, Y.J. Enhanced expression of glucose transporter-1 in vascular smooth muscle cells via the Akt/tuberous sclerosis complex subunit 2 (TSC2)/mammalian target of rapamycin (mTOR)/ribosomal S6 protein kinase (S6K) pathway in experimental renal failure. J. Vasc. Surg. 2013, 57, 475-485. [CrossRef] [PubMed] 
40. Marino, S.; Hogue, I.B.; Ray, C.J.; Kirschner, D.E. A methodology for performing global uncertainty and sensitivity analysis in systems biology. J. Theor. Biol. 2008, 254, 178-196. [CrossRef] [PubMed]

41. Hindmarsh, A.C.; Brown, P.N.; Grant, K.E.; Lee, S.L.; Serban, R.; Shumaker, D.E.; Woodward, C.S. SUNDIALS: Suite of Nonlinear and Differential/Algebraic Equation Solvers. ACM Trans. Math. Softw. 2005, 31, 363-396. [CrossRef]

42. Heldt, T. Continuous blood pressure-derived cardiac output monitoring-should we be thinking long term? J. Appl. Physiol. 2006, 101, 373-374. [CrossRef]

43. Olufsen, M.S.; Peskin, C.S.; Kim, W.Y.; Pedersen, E.M.; Nadim, A.; Larsen, J. Numerical simulation and experimental validation of blood flow in arteries with structured-tree outflow conditions. Ann. Biomed. Eng. 2000, 28, 1281-1299. [CrossRef] [PubMed]

44. Severi, S.; Cavalcanti, S.; Mancini, E.; Santoro, A. Heart rate response to hemodialysis-induced changes in potassium and calcium levels. J. Nephrol. 2001, 14, 488-496. [PubMed]

45. Selby, N.M.; Burton, J.O.; Chesterton, L.J.; McIntyre, C.W. Dialysis-induced regional left ventricular dysfunction is ameliorated by cooling the dialysate. Clin. J. Am. Soc. Nephrol. CJASN 2006, 1, 1216-1225. [CrossRef] [PubMed]

46. Schollenberger, J.; Figueroa, C.A.; Nielsen, J.F.; Hernandez-Garcia, L. Practical considerations for territorial perfusion mapping in the cerebral circulation using super-selective pseudo-continuous arterial spin labeling. Magn. Reson. Med. 2020, 83, 492-504. [CrossRef] [PubMed]

47. Debbaut, C.; Monbaliu, D.R.; Segers, P. Validation and calibration of an electrical analog model of human liver perfusion based on hypothermic machine perfusion experiments. Int. J. Artif. Organs 2014, 37, 486-498. [CrossRef]

48. Hasler, D.; Anagnostopoulou, P.; Nyilas, S.; Latzin, P.; Schittny, J.; Obrist, D. A multi-scale model of gas transport in the lung to study heterogeneous lung ventilation during the multiple-breath washout test. PLoS Comput. Biol. 2019, 15, e1007079. [CrossRef]

49. Albanese, A.; Cheng, L.; Ursino, M.; Chbat, N.W. An integrated mathematical model of the human cardiopulmonary system: Model development. Am. J. Physiol. Heart Circ. Physiol. 2016, 310, H899-H921. [CrossRef]

50. Carlson, B.E.; Secomb, T.W. A theoretical model for the myogenic response based on the length-tension characteristics of vascular smooth muscle. Microcirculation 2005, 12, 327-338. [CrossRef]

51. Arciero, J.C.; Secomb, T.W. Spontaneous oscillations in a model for active control of microvessel diameters. Math. Med. Biol. J. IMA 2012, 29, 163-180. [CrossRef]

52. Fry, B.C.; Edwards, A.; Layton, A.T. Impacts of nitric oxide and superoxide on renal medullary oxygen transport and urine concentration. Am. J. Physiology. Ren. Physiol. 2015, 308, F967-F980. [CrossRef]

53. Layton, A.T.; Edwards, A.; Vallon, V. Renal potassium handling in rats with subtotal nephrectomy: Modeling and analysis. Am. J. Physiol. Ren. Physiol. 2018, 314, F643-F657. [CrossRef] [PubMed]

54. Jackson, W.F. Ion channels and vascular tone. Hypertension 2000, 35, 173-178. [CrossRef] [PubMed]

55. Kapela, A.; Tsoukias, N.M. Multiscale FEM modeling of vascular tone: From membrane currents to vessel mechanics. IEEE Trans. Biomed. Eng. 2011, 58, 3456-3459. [CrossRef]

56. Tykocki, N.R.; Boerman, E.M.; Jackson, W.F. Smooth Muscle Ion Channels and Regulation of Vascular Tone in Resistance Arteries and Arterioles. Compr. Physiol. 2017, 7, 485-581.

57. McIntyre, C.W.; Goldsmith, D.J. Ischemic brain injury in hemodialysis patients: Which is more dangerous, hypertension or intradialytic hypotension? Kidney Int. 2015, 87, 1109-1115. [CrossRef] [PubMed]

58. Calmelet, C.; Hotchkiss, J.; Crooke, P. A mathematical model for antibiotic control of bacteria in peritoneal dialysis associated peritonitis. Math. Biosci. Eng. MBE 2014, 11, 1449-1464. [CrossRef]

59. Melis, A.; Clayton, R.H.; Marzo, A. Bayesian sensitivity analysis of a 1D vascular model with Gaussian process emulators. Int. J. Numer. Methods Biomed. Eng. 2017, 33, 25999317. [CrossRef]

60. Coveney, S.; Corrado, C.; Roney, C.H.; O’Hare, D.; Williams, S.E.; O’Neill, M.D.; Niederer, S.A.; Clayton, R.H.; Oakley, J.E.; Wilkinson, R.D. Gaussian process manifold interpolation for probabilistic atrial activation maps and uncertain conduction velocity. Philos. Transactions Ser. A Math. Phys. Eng. Sci. 2020, 378, 20190345. [CrossRef]

61. Chang, E.T.; Strong, M.; Clayton, R.H. Bayesian Sensitivity Analysis of a Cardiac Cell Model Using a Gaussian Process Emulator. PLoS ONE 2015, 10, e0130252. [CrossRef]

62. Muszkiewicz, A.; Britton, O.J.; Gemmell, P.; Passini, E.; Sanchez, C.; Zhou, X.; Carusi, A.; Quinn, T.A.; Burrage, K.; Bueno-Orovio, A.; et al. Variability in cardiac electrophysiology: Using experimentally-calibrated populations of models to move beyond the single virtual physiological human paradigm. Prog. Biophys. Mol. Biol. 2016, 120, 115-127. [CrossRef] [PubMed] 